\title{
INVESTIGACIONES
}




\section{HACIA UNA HISTORIA DE LA \\ PRACTICA PEDAGOGICA \\ COLOMBIANA}

\section{Enfoque conceptual básico del proyecto}

Existe una institución del saber que ha sido reconocida estatutariamente para difundir, velar, reproducir, investigar, reglamentar metódica y teóricamente el saber pedagógico, conformada por las Facultades de Educación y las Normales; además existe un sujeto llamado maestro, formado en las facultades para utilizar y reproducir los conocimientos y prácticas que deben efectuarse en las instituciones educativas.

La vida institucional de la Pedagogía y el maestro ha sido, hasta el momento, ignorada en nuestra historia, un silencio donde otros posan su mirada de juez para excluirla como campo de reflexión y de investigación. Se señala como pedagógico lo que corresponde a las Facultades de Educación y al maestro; se revisten de verdad otros conocimientos sin estigmas pedagógicos; se aisla la Pedagogía, su institución, y sus maestros para cuestionarla y buscar desterrarla de los territorios del saber que circula en las universidades; se olvida que todos los saberes que han circulado en la escuela son enunciados según formas o métodos pedagógicos, y en consecuencia se define como único de las Facultades de Educación y de las Normales.

Resarcir este olvido implica volcar la historia sobre el maestro y las instituciones del saber y, reescribir su historia excluida por la mirada "ilustrada" de aquellos que aún padecen la ilusión de la verdad sin reconocer que todos los conocimientos impartidos en las instituciones mediante las prácticas pedagógicas, llegan a ser solidarios con la voluntad de decir.

Aproximarse a la historia de las "forma de lo dicho" en nuestro país, es apropiarse de un capítulo de nuestra historia cultural; es convertir en objeto de análisis la memoria del saber institucionalizado y de los sujetos que lo enuncian; es describir en su funcionamiento institucional, la interioridad del discurso pedagógico, su práctica y las funciones que asigna a los sujetos seleccionados para la distribución del saber. Esto implica considerar la Pedagogía no en sí misma, sino en un complejo de relaciones. Sólo un estudio de carácter histórico puede mostrar el lugar que han ocupado las instituciones formadoras de docentes en el conjunto de las instituciones del saber de nuestra sociedad.

El enfoque elegido para el análisis debe entender aquel complejo de relaciones como una práctica. Se trata entonces de la historia de una práctica donde se busca aplicar el modelo de análisis que propone la noción de PRACTICA PEDAGOGICA.

Práctica pedagógica es una noción que designa: a) Los modelos pedagógicos, tanto teóricos como prácticos utilizados en los diferentes niveles de enseñanza. b) La pluralidad de conceptos pertenecientes a campos heterogéneos de conocimiento, retomados y aplicados por la Pedagogía. c) Las formas de funcionamiento de los discursos en las instituciones educativas, donde se realizan prácticas pedagógicas. d) Las características sociales adquiridas por la práctica pedagógica, en las instituciones educativas de una sociedad dada, que asigna unas funciones a los sujetos de esa práctica. 
La noción explicada imprime a esta investigación su objeto de análisis: LO PEDAGOGICO como teoría y como práctica; como elemento que permite a los maestros entrar en relación con el conocimiento; como acontecimiento social susceptible de ser descrito en su especificidad histérica. Se trata, pues, de seguir estas directrices para analizar nuestras prácticas pedagógicas desde la Colonia hasta el surgimiento de las Facultades de Educación.

Para delimitar lo pedagógico como teoría y como práctica, el trabajo de investigación se sitúa en la forma de existencia cotidiana de la Pedagogía: la Didáctica. La Pedagogía se apropia de múltiples nociones de otros conocimientos, para utilizarlos en diferentes procesos. Sin embargo, existe un campo específico donde aquella se convierte en un saber aplicado, que particulariza y operacionaliza. Es alrededor del proceso enseñanzaaprendizaje, como ejercicio metódico, donde se reúnen enunciados de diferentes dominios del conocimiento, individualizándose así una multiplicidad de conceptos o nociones que podemos denominar la Didáctica.

La "MANERA DE DECIR" la cual es siempre referida al conocimiento, en un proceso enseñanza-aprendizaje es llamada en Pedagogía la Didáctica. El método es asunto de aprendizaje, o sea que la manera de decir" debe ser de tal forma que el sujeto aprenda.

En el grupo de métodos que podríamos llamar tradicionales y en otros nuevos, que buscan desplazar a los primeros se encuentran problemas similares que la Didáctica ha tratado de afrontar: cómo ordenar el DECIR o la exposición en la enseñanza; a qué recurrir primero, a los objetos o a las palabras; por dónde empezar, por lo general o por lo particular; cómo conoce el hombre y acorde a esto como proceder a enseñar; cuáles conocimientos deben preceder a los otros; según la etapa de vida de quien aprende, qué debe enseñársele; en qué se diferencia el conocimiento del hombre del conocimiento científico; cuáles discursos deben seleccionarse para la enseñanza según dos necesidades: un saber para conocer y un saber para utilizar, etc. Esto no significa que el análisis será teórico; se abordará desde el punto de vista histórico, y el discurso de la Pedagogía no se reducirá al silencio.

Para que este trabajo pueda integrar su método y su objeto de conocimiento, es necesario el examen del registro histórico: el estudio del documental que haga referencia al pensamiento pedagógico en Colombia. No se entiende con esto que exista un pensamiento nacional al respecto, aún no se está en condiciones de afirmarlo o de negarlo; no obstante, lo que sí ha existido, son unas condiciones de apropiación del saber pedagógico y de otros saberes de diferentes culturas; una forma propia de enunciación, por parte de los maestros del saber pedagógico; una manera de inscribir alguna corriente pedagógica en las ideologías políticas del país; un señalamiento de las funciones sociales y económicas de la enseñanza en las prácticas educativas del país; una "dispersión" de "lo dicho" alrededor de la educación, en diferentes instituciones y prácticas; una forma de adecuar socialmente el discurso educativo y los discursos que conllevan su uso.

Se enfatizará en los métodos pedagógicos seleccionados, qué ideologías los han impulsado $\mathrm{y}$, qué redistribuciones han sufrido esos pensamientos pedagógicos al ser registrados en nuestra cultura; a qué prácticas estuvo sujeta la práctica pedagógica en el país, qué modelo o qué modelos han acogido esas prácticas pedagógicas desde el siglo $\mathrm{XVI}$ y cuándo se puede hablar de cambios en la práctica discursiva que la ha regulado.

Estas investigaciones acerca de la práctica pedagógica, se inscriben en un horizonte general que bien puede llamarse, las condiciones histórico culturales del maestro, 
condiciones que sólo podrán conocenso a través de la historia de nuestra práctica pedagógica. Pero también, un punto de ese horizonte general, es la pregunta por la función social asignada al maestro como 'agente de la cultura". Al maestro se le ha declarado como 'trasmisor de conocimientos", pero a la vez se le ha negado su acceso a la cultura.

¿Quién es el maestro? y ¿Quién entre todos los sujetos de una sociedad dada, es un maestro? En la historia encontramos el método de enseñanza, como distintivo del maestro, como si los conocimientos estuvieran hechos de una sustancia maleable y fuese preciso moldear-los, y como si el lenguaje del conocimiento tuviera que ser mirado y enunciado a través de una rejilla.

La Pedagogía no es solamente un discurso acerca de la enseñanza, sino también una práctica cuyo campo de aplicación es el discurso. El médico, por ejemplo, enfrenta sus conocimientos a la enfermedad y la forma de indagar la hace a través de unos instrumentos los cuales aplica al cuerpo; el maestro enfrenta sus conocimientos pedagógicos al discurso de las teorías o de las ciencias y el instrumento que utiliza para ello es el METODO DE ENSEÑANZA. Es esta condición del maestro (tener que adecuar el discurso de la ciencia a la edad de los sujetos del aprendizaje, hacerlo comprensible, dosificarlo y calificarlo), la que hace que su relación con el conocimiento sea a través de una práctica pedagógica.

La investigación propuesta se refiere al maestro como funcionario de la práctica pedagógica. Se refiere al ver, al hablar, al pensar y a otras funciones que involucran el oficio de enseñar, a los espacios teóricos correlativos a esas funciones, es decir, a aquellos lenguajes que le prestan la palabra a la Pedagogía y a los maestros para ejercer sus funciones pedagógicas. Se refiere, a las orientaciones que la práctica social ha impreso en la práctica pedagógica.

Para enseñar hay que decir y ese decir ha de traer a nuestra presencia el discurso transformado en cosas, en una palabra, "materializado", referido a algo que se supone concreto, designable o demostrable. Porque la Pedagogía trata los discursos como objetos. Podemos pensar que a la pareja enseñanza-aprendizaje corresponde la pareja "manera de decir"-“ manera de conocer".

Teniendo en cuenta que la relación enseñanza-aprendizaje implica dos sujetos: el que dice y el que conoce, señalemos el interés de la Pedagogía en esta afirmación: Según el decir es el aprender. Precisamente por ser "el decir" lo que fundamenta la relación del maestro con el conocimiento, la Pedagogía suministra aquellos instrumentos a la formación de maestros que dominen "el decir". Es casi secundario que se conozca suficientemente un saber; si se sabe decir, se puede pronunciar, o se puede describir y hasta demostrar, a la vista de quien aprende, cualquier discurso. La palabra hablada describe y demuestra, hace la verdad en el salón de clase.

Quien enseña, sabe decir, esto y define su estatus de maestro, además está investido del poder que le confiere una institución; el poder de decir, de pronunciar los discursos que ésta regula. En este sentido quien enseña ejerce el poder por medio del discurso. La asimilación del discurso es medible, por tanto, aquello) que se dice conlleva unas características de tal índole que hacen posible la medición. El decir del profesor pone no sólo junto al oído sino también y, fundamentalmente ante la vista del sujeto que aprende, los enunciados que habrá de apropiarse: enunciados palpables, captables, aprehensibles, evidentes, familiares, reducidos a la máxima expresión de la simpleza, a la posibilidad más cercana de aplicación, de apreciación, de ejemplarización, de discusión, enunciados 
casi materiales. EL DECIR DEL PROFESOR MATERIALIZA LOS ENUNCIADOS. Es necesario) que la "manera de decir", como) mandato de la Didáctica, se reduzca a lo mas simple, materialice la palabra, haga de los discursos situaciones predicables y forme la pareja ver-hablar.

La pareja ver-hablar es una condición del conocimiento, condición básica de los supuestos acerca del mismo, sobre los cuales descansa la Didáctica. Enseñar es producir el acontecimiento donde entran en escena, un sujeto que aprende, un sujeto que enuncia y un objeto que surge del decir del sujeto enunciante.

Podría preguntarse: ¿un objeto? ¿Qué surge del decir del sujeto enunciante? Ante la exigencia de la simpleza, de la claridad expositiva, de la necesidad de asimilación del sujeto que aprende, la Pedagogía trata los discursos como objetos. Es aquí donde apareció la posibilidad de esta investigación y es aquí donde se vuelve para continuar el camino.

Precisamente por haber asumido que la Pedagogía no es solamente un discurso acerca de la enseñanza, sino también una práctica cuyo campo de aplicación es el discurso, ha sido fundamental para este enfoque problematizar la relación Pedagogía-Lenguaje para orientar luego el trabajo histórico sobre los nexos que mantuvo la Pedagogía, en nuestra sociedad, con un conjunto de saberes que asignaba al lenguaje funciones utilizadas por la práctica pedagógica.

El proyecto relieva el ser histórico de la Pedagogía, ya que todo saber ocupa un espacio histórico en la práctica pedagógica, espacio definido por el régimen cultural del conocimiento en toda sociedad.

Buscando difundir y fomentar el interés por la historia de la cultura, que ha pasado por nuestra práctica pedagógica y, por el análisis de las funciones asignadas a los sujetos de esta práctica, se inició un trabajo conjunto entre la Universidad Pedagógica Nacional, Universidad del Valle, Universidad Nacional de Colombia (sede Bogotá) y Universidad de Antioquia, acogiendo del proyecto "Filosofía y Pedagogía" ${ }^{22}$ su enfoque, su metodología, su noción de práctica pedagógica y sus problematizaciones en la forma como la Pedagogía trata los discursos a través de los diferentes procesos educativos colombianos.

\section{Objetivos}

\section{Objetivos generales}

- Promover un proceso de investigación que permita asumir el ARCHIVO PEDAGOGICO del país, como un objeto de análisis para fines académicos e investigativos en las Facultades de Educación.

-Asumir el discurso de la Pedagogía como un complejo de relaciones entre discursos (provenientes de diferentes dominios del conocimiento) y prácticas sociales que regulan el saber y su ejercicio institucional.

\footnotetext{
${ }^{122}$ Proyecto del Centro de Investigaciones Educativas. Facultad de Educación. Universidad de Antioquia. Dirigido por la profesora Olga Lucia de Echeverry.
} 
—Describir históricamente las relaciones entre prácticas o contenidos pedagógicos, según los sistemas de relaciones que han regido el saber pedagógico como práctica discursiva en nuestro país.

-Establecer las particularidades de los contenidos pedagógicos que han circulado por nuestras prácticas pedagógicas.

- Ubicar en el archivo pedagógico del país indicadores de grupos de relaciones, susceptibles de ser individualizados en estudios posteriores.

\section{Objetivos específicos}

- Caracterizar las modalidades pedagógicas que han regido el ejercicio de la enseñanza en nuestra escuela y la relación del maestro con el conocimiento.

- Describir la transformación del contenido y de la práctica pedagógica, según relaciones establecidas con el conjunto de conocimientos que le han apoyado teórica, metódica e instrumentalmente a través de la historia.

- Comparar las diversas modalidades pedagógicas impartidas en las prácticas e instituciones que se han ocupado del saber pedagógico.

-Describir los enunciados que han fundamentado las concepciones del conocimiento, del método, del hombre y del maestro en las modalidades pedagógicas de la Colonia, en las Normales y Facultades de Educación.

- Relacionar las formas pedagógicas utilizadas en la enseñanza impartida en la lengua indígena, latina o castellana desde la evangelización, como primer proceso pedagógico, hasta la aparición de las Facultades de Educación.

- Relacionar y comparar los momentos de transformación o de permanencia de las relaciones entre contenidos y prácticas pedagógicas, asumiendo como directrices los contenidos pedagógicos y las finalidades sociales asignadas a las prácticas pedagógicas.

- Inventariar y reseñar a lo largo del desarrollo del proyecto, otras posibilidades de análisis del archivo pedagógico del país según diferentes sistemas de relaciones articulables.

\section{Carácter interinstitucional del proyecto}

\section{Hipótesis matriz del proyecto}

La hipótesis matriz del proyecto es la relación que el método pedagógico establece entre conocimientos y lenguaje y la condición social de la Pedagogía como práctica.

\section{La complejidad del objeto a investigar}

De la Colonia hasta el surgimiento y la evolución de las Facultades de Educación es apenas una afirmación, si se tiene en cuenta que la unidad del proyecto general no está fundamentada ni en un punto inicial, ni en un punto final cronológico. Se trata de seguir 
históricamente las transformaciones de la práctica docente y de las instituciones que han acogido un saber designado Pedagogía, unas formas específicas de ejercerlo y unas relaciones en el conjunto de instituciones del saber a las cuales han estado socialmente articuladas.

Los objetivos del proyecto han considerado la necesidad de inventariar durante su ejecución, otras posibilidades de análisis u otras particularidades de nuestra práctica pedagógica, que sirvan de base a estudios regionales de conjuntos articulables.

\section{El enfoque conceptual y metodológico}

La forma de asumir el documento como un registro de relaciones múltiples, exige su lectura atenta y una paciente labor de tematización, punto básico para seleccionar las temáticas directrices del proyecto general. Esto crea la permanente necesidad de discutir, comparar, relacionar, diferenciar los posibles Conjuntos de temáticas y las particularidades que vayan marcando cortes para periodizar.

\section{CUADR01}

\section{INTERRELACIÓN DE LOS PERIODOS DE LOS SUBPROYECTOS}

\begin{tabular}{|c|c|c|c|}
\hline $\begin{array}{l}\text { La practica } \\
\text { pedagógica de } \\
\text { La colonia }\end{array}$ & $\begin{array}{l}\text { Los jesuitas como } \\
\text { maestros }\end{array}$ & $\begin{array}{l}\text { La practica pedagógica del } \\
\text { siglo XIX }\end{array}$ & $\begin{array}{l}\text { La practica pedagógica } \\
\text { del siglo } X X\end{array}$ \\
\hline $\begin{array}{c}\text { Universidad } \\
\text { Pedagógica Nal. }\end{array}$ & $\begin{array}{l}\text { Universidad } \\
\text { Nacional }\end{array}$ & Universidad de Antioquia & $\begin{array}{c}\text { (Formación de docente } \\
\text { en normales y Fac. de } \\
\text { Educa.) } \\
\text { Universidad del Valle }\end{array}$ \\
\hline $\begin{array}{l}1550 \text { Escuela de } \\
\text { primeras letras }\end{array}$ & $\begin{array}{l}1598 \text { llegada de la } \\
\text { compañía de Jesús }\end{array}$ & $\begin{array}{l}1826 \text { Plan de estudios de } \\
\text { Santander. Sistema de } \\
\text { enseñanza mutua }\end{array}$ & $\begin{array}{l}1886 \text { La constitución } \\
\text { nacional }\end{array}$ \\
\hline $\begin{array}{l}1654 \text { Colegio Mayor } \\
\text { del Rosario }\end{array}$ & $\begin{array}{l}1767 \text { Primera } \\
\text { expulsión }\end{array}$ & $\begin{array}{l}1842 \text { La reforma Alcántara } \\
\text { Herrán }\end{array}$ & 1903 La reforma Uribe \\
\hline $\begin{array}{l}1774 \text { Plan Moreno y } \\
\text { Escandón }\end{array}$ & $\begin{array}{l}1843 \text { Segunda } \\
\text { Ilegada }\end{array}$ & 1850 Libertad de enseñanza & 1913 El Gimnasio Moderno \\
\hline \multirow[t]{4}{*}{$\begin{array}{l}1783 \text { Mutis y la } \\
\text { Expedición Botánica }\end{array}$} & $\begin{array}{l}1850 \text { Segunda } \\
\text { expulsión }\end{array}$ & $\begin{array}{l}1870 \text { Sistema de enseñanza } \\
\text { Pestalozziana }\end{array}$ & $\begin{array}{l}1950 \text { La tecnología } \\
\text { educativa }\end{array}$ \\
\hline & 1858 Tercera llegada & 1886 La constitución nacional & $\begin{array}{l}1966 \text { tercer seminario } \\
\text { facultades de educación }\end{array}$ \\
\hline & $\begin{array}{l}1861 \text { Tercera } \\
\text { expulsión }\end{array}$ & 1887 El concordato & \\
\hline & & 1903 La reforma Uribe & \\
\hline
\end{tabular}

La dispersión de la documentación y la escasez de material documental auxiliar, requiere aunar esfuerzos para aprovechar al máximo la fuerza de trabajo intelectual y aprovechar conjuntamente las fuentes para el trabajo de los subproyectos durante períodos similares.

La noción de práctica pedagógica como noción articuladora, tanto teórica como metodológicamente, de los diferentes frentes de la investigación exige la caracterización de series diferenciables pero en relación unas con otras; la permanencia o transformación de esas relaciones sólo podrá establecerse por el trabajo de un equipo conocedor de las implicaciones metodológicas de la noción de práctica pedagógica y, por consiguiente de 
los nexos que el trabajo documental de cada frente guarde con el proyecto general para efectos comparativos y con el propio para fines descriptivos.

\section{Interrelación de los períodos de los subproyectos}

La magnitud del objetivo que el proyecto interuniversitario se ha propuesto, -iniciar el acercamiento a la pedagogía en Colombia - requiere de una periodización que marque cortes y unidades, en esa historia, tratada tradicionalmente como un continuo jalonado por hitos jurídicos o políticos pero que no da cuenta de la autonomía relativa y específica de los desarrollos que la práctica pedagógica del país ha sufrido.

Sin embargo, la periodización actual que el proyecto ha establecido se basa en dos criterios:

— La aceptación provisional de esos cortes que la historia tradicional ha marcado, sólo como puntos de referencia cronológica que distinga unos períodos de otros: colonia, primeros años de vida independiente, reformas liberales, federalismo, regeneración y época actual.

— La orientación teórica y metodológica del proyecto piensa una periodización basada en el restablecimiento de unidades, a partir de las coyunturas vistas en las transformaciones registradas en la práctica pedagógica nacional, tal como el proyecto la ha definido. Pero esa periodización, será un resultado.

Por tanto, los fines de la periodización propuesta para el proyecto, tienen un carácter operativo, es decir, delimitar para cada subproyecto los limites de la exploración documental, de acuerdo a los temas y posibilidades de recursos humanos y materiales de cada grupo de trabajo, y para funcionalizar el seguimiento de las relaciones temáticas planteadas en esos mismos subproyectos (véase cuadro de periodizaciones).

\section{El mejoramiento cualitativo de las instituciones formadoras de docentes}

El análisis de la formación de docentes en nuestra sociedad, debe ser investigado históricamente, si se busca dar una solución a los problemas que se debaten en la actualidad acerca de las Facultades de Educación y en general a la formación de docentes.

La orientación pedagógica en las Facultades de Educación está todavía enraizada en una pedagogía tradicional que concibe el conocimiento de manera operativa y normativa; el maestro debe proceder de tal o cual forma en la enseñanza y debe cumplir con una reglamentación asignada a su oficio.

Tres instituciones han formado maestros en nuestra historia: la Iglesia durante la Colonia, las Normales que surgen durante el siglo XIX y las Facultades de Educación fundadas durante el siglo XX. Son las Normales las primeras instituciones del saber pedagógico y por tanto formadoras de un sujeto diferenciado y reconocido como tal mediante ese saber; pero éstas nacieron inscritas en diversos conjuntos de relaciones entre el saber pedagógico y otros conjuntos de saberes heredados de la Colonia que pugnaban por parecer o predominar en la práctica pedagógica colombiana. La diferencia entre el maestro formado en el pensamiento Colonial y el maestro formado por las Normales, es posible establecerla, si se piensa desde la institución, su saber, el sujeto de ese saber y su práctica. Sólo así se podrá analizar la permanencia, la diferencia o la separación entre estas instancias en los diferentes períodos de la historia de la Pedagogía. No puede 
hablarse de cortes totales; bien conocido es que la influencia de la Iglesia en la cultura y la enseñanza se vio sometida a una serie de discontinuidades que pusieron en entredicho su hegemonía cultural y pedagógica durante el siglo XIX.

Corresponde, pues, a la historia, la palabra, para mostrar el carácter de nuestras instituciones formadoras de docentes; los resultados del proyecto propuesto contribuirán a un diagnóstico de la concepción pedagógica por ellas difundida y practicada.

Aunque en la actualidad se enseñe a los maestros teorías pedagógicas modernas, éstas siguen coexistiendo con una práctica pedagógica de carácter tradicional que se ha convertido en un obstáculo de conocimiento, tanto para la formación del maestro, en las teorías o ciencias que le corresponderá enseñar, como para una formación pedagógica del más alto nivel. La forma de existencia del saber pedagógico en nuestro país, no ha acogido los desarrollos de la pedagogía como disciplina que busca un estatuto teórico y aplicado, apoyándose en avances de otros campos del conocimiento. Por tanto, carecemos de instituciones formadoras de docentes capaces de apropiar social y culturalmente modelos pedagógicos, capaces de infundir, investigar y experimentar un saber pedagógico de la más alta calidad que supere los estrechos marcos de la pedagogía tradicional.

El proyecto relieva el ser histórico de la pedagogía, ya que todo saber ocupa un espacio histórico en la práctica pedagógica, espacio definido por el régimen cultural del conocimiento en toda sociedad.

Los resultados del proyecto pueden incidir en una reorientación de las instituciones formadoras de docentes que reconozca la pedagogía como campo específico dotado de objetos de conocimiento, y de un campo conceptual para la observación, la experimentación y la investigación. Esta última, entendida en un doble sentido: desde el interior de su teoría y desde el interior de su práctica en las instituciones del saber.

La Pedagogía como una forma de relación con el conocimiento, la historia de la pedagogía concebida como historia de la cultura, ha sido ignorada en nuestro país, a tal punto que los documentos, donde quedó registrada nuestra práctica pedagógica, han sido considerados marginales. Se trata en el análisis propuesto de rescatar documentos ignorados y olvidados: EI ARCHIVO PEDAGOGICO COLOMBIANO.

Finalmente, es de resaltar la importancia de una cultura documental para el maestro que le haga comprender el valor histórico y nacional del registro pedagógico, el valor cultural de la enseñanza. Formar maestros conscientes de la historia pedagógica colombiana, hacer que nuestras instituciones formadoras de docentes asuman el archivo pedagógico colombiano para fines académicos e investigativos, sería propender por la creación de una intelectualidad, capaz de utilizar como herramienta de producción del saber pedagógico, observaciones, conceptualizaciones y experimentaciones de otros campos del saber.

Olga Lucía de Echeverry 